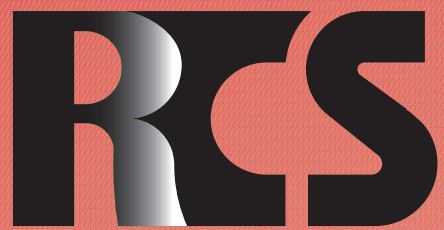

Depósito legal ppi $201502 Z U 4662$

Esta publicación científica en formato digital es continuidad de la revista impresa Depósito Legal: pp $197402 Z U 789$

- ISSN: 1315-9518 • ISSN-E: 2477-9431

Revista de Ciencias Sociales

Universidad del Zulia. Revista de la Facultad de Ciencias Económicas y Sociales Vol. XXVII. No. 2

Abril-Junio 2021

Esta publicación científica en formato digital es continuidad de la revista impresa Depósito Legal: pp $197402 Z$ Z789 ISSN: 1315-9518 


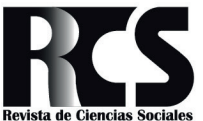

Revista de Ciencias Sociales (RCS). FCES - LUZ

Vol. XXVII, No. 2, 2021, pp. 199-216 Abril-Junio

- ISSN: 1315-9518 IISSN-E: 2477-9431
Como citar APA: Villasmil, M. del C., Romero, F., y Socorro, C. C. (2021). Liderazgo ético en la gestión pública municipal del estado Zulia, Venezuela. Revista de Ciencias Sociales (Ve), XXVII(2), 199-216.

\title{
Liderazgo ético en la gestión pública municipal del estado Zulia, Venezuela
}

\author{
Villasmil, Milagros del C." \\ Romero, Francisco J." \\ Socorro, Cecilia C.**
}

\section{Resumen}

Para que las administraciones públicas sean éticas en el desempeño de su gestión, las decisiones de sus representantes deben responder a los intereses de los grupos de interés a los que se encuentra vinculada, además sus funciones deben ser ejercidas con voluntad de servicio público. En relación a ello, la presente investigación analiza el liderazgo ético en la gestión pública municipal, buscando potenciar la eficiencia en el desempeño de sus funciones públicas con responsabilidad social; enmarcada en el paradigma cualitativo, etnográfico, interpretativo, de carácter transversal, cuya población de estudio fue la Alcaldía de Mara del estado Zulia, Venezuela. Donde se evidenció que se han intentado desarrollar cambios científicos-sociales, sin embargo, a pesar de los esfuerzos realizados por alcanzar la trasformación organizacional, existen algunas limitaciones desde el punto de vista político, jurídico, económico, social y tecnológico por lo cual, se ha establecido una propuesta del liderazgo ético como factor potenciador de la transformación organizacional, en base a las características y perfil del líder. Se concluye, que dichas modificaciones deben canalizarse de manera metódica, induciendo a los involucrados, a través de talleres grupales, a un cambio gradual progresivo, que permitan generar el cambio necesario en la administración pública municipal del estado Zulia.

Palabras clave: Liderazgo; ética; gestión pública; transformación organizacional; responsabilidad social.

\footnotetext{
* Doctora en Ciencias Gerenciales. Magister en Gerencia Tributaria. Licenciada en Contaduría Pública. Docente e Investigadora en la Universidad Libre Seccional Barranquilla, Colombia. Investigadora de REOALCeI (Gestión Contable, Financiera, Tributaria y Aseguramiento). Vinculada al Grupo de Investigación Tendencias Contables, Económicas y Administrativas TCEA, categorizado en A. E-mail: milagrosd.villasmilm@unilibre.edu.co iD ORCID: https://orcid.org/0000-0002-8222-8871

** Doctor en Ciencias Gerenciales. Especialista en Auditoria Financiera. Contador Público. Docente Titular e Investigador del Sector Universitario. Adscrito al Decanato de Post Grado de la Universidad Dr. Rafael Belloso Chacín (URBE), Maracaibo, Venezuela. E-mail: fjromero@urbe.edu.ve iD ORCID: https://orcid.org/0000-0003-3994-014X

*** Doctora en Ciencias Gerenciales. Magister en Gerencia Financiera. Economista. Profesora e Investigadora de la Universidad del Zulia (LUZ), Venezuela. E-mail: mgs.cecri.fces@gmail.com iD ORCID: https://orcid.org/0000- 0002$\underline{4682-6102}$
}

Recibido: 2020-12-11

Aceptado: 2021-02-26 


\title{
Ethical leadership in municipal public management in the state of Zulia, Venezuela
}

\begin{abstract}
For public administrations to be ethical in the performance of their management, the decisions of their representatives must respond to the interests of the interest groups to which they are linked, in addition their functions must be exercised with the will of public service. In relation to this, this research analyzes ethical leadership in municipal public management, seeking to enhance efficiency in the performance of its public functions with social responsibility; framed in the qualitative, ethnographic, interpretive, crosssectional paradigm, whose study population was the Mayor's Office of Mara in the state of Zulia, Venezuela. Where it was evidenced that attempts have been made to develop scientific-social changes, however, despite the efforts made to achieve organizational transformation, there are some limitations from the political, legal, economic, social and technological point of view, therefore, has established a proposal for ethical leadership as a factor that enhances organizational transformation, based on the characteristics and profile of the leader. It is concluded that said modifications must be channeled in a methodical way, inducing those involved, through group workshops, to a gradual progressive change, which allows generating the necessary change in the municipal public administration of the state of Zulia.
\end{abstract}

Keywords: Leadership; ethics; public management; organizational transformation; social responsibility.

\section{Introducción}

A nivel mundial, el liderazgo ético ha sido analizado por expertos desde un enfoque científico-social, por cuanto los observadores han aludido durante mucho tiempo a los rasgos personales de la integridad ética, el carácter, el altruismo, la motivación colectiva, la conciencia ética, como indispensables para el liderazgo ético confiable en la organización.

En tal sentido, los líderes son considerados como personas justas, con unos principios éticos coadyuvantes a la toma de decisiones para el bienestar común (Mendoza y Mendoza, 2008). Al respecto, Brown y Treviño (2006), han definido el liderazgo ético como generador de una relación positiva con la confianza afectiva en el líder, más que una relación negativa con la supervisión abusiva, que no está relacionada con la similitud percibida entre el líder y el subordinado.

El comportamiento del líder, según Paz, et al. (2016), comprende el principio de su actuación social, el cual procede de la voluntad de su acción frente al bien o el mal, es decir, parte de los aspectos acerca de lo que se hace, o también de aquello que se dice, resultando imprescindible actuar no solo de forma responsable sino a su vez de manera transparente y con sentido ético.

En los actuales momentos políticosociales de Venezuela, donde si bien se requieren conductores de individuos centrados en la ética, debe abocarse su perfil a la visión transformacional. En el caso de la administración pública, Espinal (2017), plantea que es necesario un líder defensor, con carácter axiológico, valorativo, internalizando su condición ciudadana y de servidor público, debe practicar un modelaje congruente; apoyarse en reglas secuenciales, sistemas laborales lógicos, procedimientos, controles, cuando resulte necesario, evitando la burocracia, el formalismo excesivo, que responda a las demandas sociales con eficacia, eficiencia, procurando incrementar sus niveles de calidad en un contexto servicial, para la gente.

Sin embargo, tal como lo expresan Guaiquirima y Seijo (2010), el liderazgo ético en la gestión pública juega un papel determinante en dicha gestión, sobre todo en la creación de un clima moral, agradable, armónico, esencial para la supervivencia 
social. No obstante, la problemática existente dentro de las organizaciones públicas, radica en la necesidad de recuperar la ética como valor fundamental en la actuación de los funcionarios que laboran en dichas instituciones, pues durante los últimos años, se ha observado cómo el desempeño de los mismos, especialmente en el municipio Mara, bajo el gobierno de Luis Caldera, responde más a lineamientos partidistas o intereses particulares, que a la satisfacción de necesidades sociales, para lo cual fueron electos, faltando así a los principios éticos y morales de su ejercicio profesional.

A su vez, consideran Calderón, Cuartas y Álvarez(2009), el liderazgo ético es un factor de éxito organizacional, pero este debe adaptarse al contexto global actual, debiendo poseer capacidad para cambiar apropiadamente, adecuarse a la transformación, esto es, desarrollar la capacidad modificadora de organizaciones estructuralmente, pero también replanteándose elementos más profundos, como valores, comportamiento, cultura, métodos operativos, manteniendo una actitud renovadora, con aprendizaje permanente.

Por lo cual, en Venezuela, según los autores Loaiza y Pirela (2015), las organizaciones periódicamente se han visto en la necesidad de replantear sus estilos de liderazgo y sus prácticas gerenciales, debiendo canalizar acciones, coyuntura derivada por las limitaciones político-económicas, las debilidades socio-culturales existentes, que impiden resultados eficientes aprovechables en pro del colectivo común.

En función a lo anterior, se pretende en este trabajo analizar el liderazgo ético en la gestión pública municipal del estado Zulia, en Venezuela, buscando potenciar la eficiencia en el desempeño de sus funciones públicas con responsabilidad social. Ello, porque se hace necesario un cambio fundamental en las formas y en los procesos gubernamentales en el ámbito local, para mejorar las estrategias innovadoras conducentes al desarrollo, el crecimiento organizacional, aumentando sus capacidades y oportunidades, favoreciendo el mejoramiento en las condiciones de vida en un municipio, solventando sus necesidades como función primordial para lo cual se crean las entidades públicas.

\section{Fundamentos teóricos}

\subsection{El liderazgo ético en el ejercicio de la función pública}

Para Romero (2006), el sentido de la ética merece destacarse como pilar fundamental dentro de las organizaciones, porque el hombre debería estar decidido a conducirse de forma oportuna a su conciencia moral, fruto de la cultura del ser y satisfacción de los buenos hábitos, en cualquier ámbito, preservando el código de conductas que normatice el cumplimiento formal de la calidad del servicio.

Por su parte agregan, Villasmil, Alvarado y Socorro (2019), que el resurgimiento de la preocupación no sólo por el tema ético sino por incluir, códigos éticos en la función pública, se debe al hecho que los modelos tradicionales de administración correspondieron a modelos políticos de tradición liberal entrando en franco desgaste, generando como consecuencia severos procesos de deslegitimación ética, política y jurídica. Esto contribuyó a generar una opinión negativa, así como también un juicio desfavorable sobre los funcionarios que vieron paulatinamente minado su prestigio.

Ahora bien, el liderazgo ético, se define como el ejercicio de las competencias básicas individuales de gestión en consonancia con los principios morales, con el propósito de fomentar y promover un ambiente ético en la organización, tal como lo describen Mendonca y Kanungo (2009), es un desempeño individual basado en la moral, que contribuye a la transformación de los seguidores, engendrando la virtud, a través del ejemplo y la conducta virtuosa del líder.

Asimismo, el liderazgo ético se observa como una conjugación de acciones personales y relaciones interpersonales; a la vez, se visualiza como un constructo ambiguo en 
Villasmil, Milagros del C.; Romero, Francisco J. y Socorro, Cecilia C.

Liderazgo ético en la gestión pública municipal del estado Zulia, Venezuela

la administración pública. Tal es el criterio de Guaiquirima y Seijo (2010), quienes afirman que el liderazgo se ejerce desde y hacia las personas. Si se prescinde de esta primera premisa se olvida lo que, realmente, es importante: Las personas son la clave de la empresa. A lo cual, agregan Alvis, Alvis y Alvis (2019), que el liderazgo es la manera específica de dirigir las actividades laborales de los miembros de un grupo y de conducirlas hacia determinados objetivos, disponiendo para ello de las herramientas establecidas por las relaciones humanas.

Bass y Bass (2008), define el liderazgo como una interacción entre miembros de un grupo, que involucra la estructuración o reestructuración de situaciones, percepciones y expectativas de los miembros. El liderazgo ocurre cuando un miembro del grupo modifica la motivación o competencias de otros o cuando direcciona su atención a las metas o al camino para alcanzarlas.

Asimismo, según Fernández y Quintero (2017), el liderazgo se caracteriza por mantener relaciones positivas con los grupos de trabajo, influyendo positivamente en el desempeño del empleado y éxitos en los objetivos organizacionales, generando confianza y cooperación en las personas que los rodean. A lo cual, agregan Correa, Rodríguez y Pantoja (2018), el liderazgo se considera como una relación de influencia que se da entre el líder y sus seguidores, mediante la cual ambas partes buscan el logro de cambios y propósitos compartidos.

En la gestión pública según VillasmilMolero, et al. (2018), la seguridad social óptima, el aumento de la calidad de vida y el desarrollo pleno del ser humano, constituyen una serie de obligaciones que el Estado debe cumplir y gestionar el empleo de los recursos públicos dirigidos a la consecución, coordinación y armonización de los planes y programas, de conformidad con el proyecto nacional.

Para la presente investigación se tomará como fundamento lo planteado por Vegas (2016), sobre la teoría fundamentada que sirve como estrategia de análisis de información empírica en el área administrativa y gerencial, específicamente dentro de la gestión pública local, ayudando a comprender mejor el fenómeno que se presenta en materia de transformación, modernización administrativa del Estado, incluso de los procesos de participación, descentralización y democratización de los servicios públicos.

Por lo tanto, para los investigadores del presente artículo el liderazgo ético en el ejercicio de la función pública, debe ser capaz de asumir y defender valores internalizados a su condición de ciudadano y al mismo tiempo de servidor público, siendo especialmente cuidadoso de ser coherente entre lo que dice y lo que hace. En otras palabras, practicar un modelaje congruente; apoyarse poco en reglas, sistemas, procedimientos y controles, acostumbrándose a vivir con un notable grado de riesgo trazado a partir de las necesidades de la comunidad; y sobre todo, una de las características esenciales de los verdaderos líderes, la generación de credibilidad y confianza que garantice la transparencia institucional.

En este sentido, conforme a lo planteado por los autores consultados, los investigadores del presente artículo infieren que dentro de la alcaldía de Mara del estado Zulia (Venezuela), se requiere de un líder con habilidad de comprender las situaciones referidas a la escasez de recursos, sabiendo distribuir los mismos, atendiendo y resolviendo las exigencias de las comunidades además de los requerimientos de la propia institución gubernamental.

Pues la contextualidad que se vive en dicha alcaldía, aunada a la propia situación económica del país y la personal de los funcionarios públicos que laboran en dicha entidad, conllevan a generar emociones en el ser humano, como respuesta a eventos producidos por las limitaciones que pueda tener dentro de su propia gestión tales como: Insatisfacción en la comunidad, falta de presupuestos para atender las necesidades sociales, negligencia por parte de los responsables de políticas públicas, carencia de valores, entre otras; donde dichas emociones 
podrían afectar la toma de decisiones del líder, desviar los intereses, y por ende el logro de los objetivos de la organización.

\subsection{Características del líder ético}

Desde un enfoque científico-social, según lo afirmado por Nava, Seijo y Mier (2014), se ha creído durante mucho tiempo que los rasgos personales tales como la integridad ética, el carácter, el altruismo, motivación colectiva, y la conciencia ética, son indispensables para un liderazgo ético confiable en la organización. Por su parte, los líderes son considerados como personas justas, con unos principios éticos que los ayudan a tomar decisiones para el bienestar común de la gente. En este marco de ideas, el liderazgo ético genera una relación positiva con la confianza afectiva en el líder y una relación negativa con la supervisión abusiva, pero no está relacionada con la similitud percibida entre el líder y subordinado (Ferrer, Colmenares y Clemenza, 2011).

Por su parte, Guaiquirima y Seijo (2010), afirman que el líder ético sabe que la credibilidad es el camino más corto entre lo que se manifiesta de boca a los demás miembros del equipo y lo que realmente se ejecuta, en otras palabras, apoya su liderazgo en resultados concretos y no en palabras, la coherencia en sus actuaciones es la columna vertebral de la confianza y la credibilidad, teniendo su pilar en la honradez, el líder ético es honrado y, además, lo parece.

A lo anterior, agregan Narváez, et al. (2009), que las empresas líderes en los diferentes mercados, basan sus modelos gerenciales en considerar primordialmente el entorno competitivo donde se desenvuelven, además que desarrollan capacidades para detectar las nuevas tendencias en los patrones de conducta de los consumidores y garantizando la comercialización de productos o servicios de alta calidad en función de esas nuevas necesidades, sin olvidar que sus actuaciones deben fundamentarse en los principios de responsabilidad, ética, cumpliendo con las reglamentaciones sociales, permitiendo un desarrollo económico y sustentable de la empresa.

A modo de reflexión, los autores de este articulo señalan entre las cualidades que deberían caracterizar el comportamiento de un líder ético, las siguientes: a) Exige resultados; b) concede un margen de maniobra a los seguidores; c) es ejemplar en todas sus actuaciones; d) delega tareas a sus colaboradores; e) se preocupa de forma constante por el desarrollo humano de sus ayudantes; f) es veraz en todo lo que comunica; g) es humilde, pues nunca se circunscribe en el error de otro y no tiene problemas en asumir que se ha equivocado, si eso sucede; h) es leal, pues nunca habla mal de ninguna persona a sus espaldas; i) exige que cualquier conflicto interpersonal se solucione; $\mathrm{y} \mathrm{j}$ ) se muestra a sus colaboradores en todo momento como prescindible, de tal forma que si se fuera ahora, la estructura seguiría siendo autosuficiente a corto plazo.

En virtud de ello, agregan los autores de este artículo, en base a lo planteado por los otros autores consultados que el liderazgo se muestra como el arte de relacionarse constructivamente con otras personas y lograr que éstas se movilicen para alcanzar determinados objetivos. Esa relación, interactiva y dinámica, está basada principalmente en sentimientos, valores y objetivos compartidos.

Siendo las mencionadas relaciones las características a ser estudiada en la presente investigación y analizando cómo se desarrollan dentro de la Alcaldía de Mara del estado Zulia (Venezuela), considerando que no se debe confundir el liderazgo con un cargo administrativo o una posición jerárquica dentro de la organización, tampoco es un título nobiliario que se adquiere una vez y se ostenta toda la vida, parámetros que los funcionarios de la mencionada entidad quizás han asumido desvirtuándolos de sus verdaderas funciones.

\subsection{El liderazgo transformacional}

En las últimas décadas, según Ramírez 
Villasmil, Milagros del C.; Romero, Francisco J. y Socorro, Cecilia C.

Liderazgo ético en la gestión pública municipal del estado Zulia, Venezuela

(2012), las diferentes organizaciones tanto públicas como privadas, se sienten obligadas a involucrarse en procesos de transformación; basadas en la necesidad de encontrar nuevas perspectivas de negocios cuando se generan rupturas del equilibrio dinámico de la organización con su entorno, o a causa de la presión de los mercados, la competencia, las medidas y políticas gubernamentales, los efectos de la globalización sobre la identidad cultural, y la creciente necesidad de flexibilidad en sus actividades, llevándolas a reajustar sus estrategias, con el objetivo de generar respuestas eficientes para responder a las diferentes demandas sociales.

Asimismo, Calderón, et al. (2009), señalan que para desarrollar el poder individual del profesional así como el poder colectivo de los miembros que laboran dentro de una organización, se precisa una transformación de la misma que permita mantener acciones y condiciones propicias para renovar, innovar $\mathrm{y}$ aprender en función a las características del entorno así como su evolución, a fin de optimizar las relaciones con los miembros tanto internos como externos de la organización.

No obstante, los esfuerzos aislados que realiza la dirección son insuficientes para generar transformaciones, dado que una vez detectada la necesidad de cambiar, la organización tiene dos alternativas: Puede intentar el uso de medidas fragmentarias derivadas de los aciertos en el pasado (implantar cambios), o bien iniciar un proceso de transformación para ajustarse a un modelo totalmente nuevo (Font, et al., 1999).

Tales procesos de transformación organizacional, de acuerdo a Petit (2012), dada su profundidad, tienen la propiedad de abarcar toda la dependencia donde se inicien esos cambios, afectando diversos aspectos dentro de la misma, tales como la estructura, la estrategia, el estilo directivo, la cultura, el ambiente, los valores, las políticas y la tecnología. Los mismos resultan necesarios aplicarlos en el actual siglo XXI, pues los enfoques de desarrollo organizacional tradicional, no permiten dar respuesta oportuna ni eficiente a los nuevos escenarios sociales y económicos emergentes, obligando a reestructurar los modelos de gestión para alcanzar el desarrollo humano y social de la nación.

En este sentido, para González y Rada (2017), el líder transformacional, debe propiciar acciones de cambios y reorientación de los procesos que se llevan a cabo con la finalidad de generar estrategias, que conlleven a la innovación en los procesos administrativos, adaptados a las nuevas tecnologías y a los cambios sociales que se viven a nivel mundial, donde dicha gestión innovadora es la que direccionará las transformaciones que permitan aportar soluciones eficaces y eficientes a las distintas comunidades.

Por lo cual, según Pedraja-Rejas, Rodríguez-Ponce y Rodríguez-Ponce (2008), establecen que el liderazgo transformacional es el medio que permite unificar intereses de los seguidores, con los de las organizaciones, generando una visión prospectiva de amplio alcance para todos los involucrados, que dirija los planes y proyectos al beneficio grupal, basados en los principios de responsabilidad social y ética, transformando realidades, en situaciones de desarrollo y bienestar.

A modo de reflexión, los autores del presente artículo manifiestan que el poder afrontar los procesos de transformación organizacional dentro de la Alcaldía de Mara, como una situación de renovación y/o ampliación corporativa, con visión prospectiva, abrirse y permitir la flexibilidad ante nuevos cambios, es lo que producirá los efectos deseados en relación al mejoramiento de sus procesos administrativos, calidad de servicio, menos burocracia, optimización del tiempo para empleados como para la población que requiere solventar algún requerimiento ante esta dependencia, produciendo un mayor impacto socio-económico en el municipio y sumando seguidores a su gestión administrativa, mejorando a su vez, las aptitudes de los miembros de la entidad pública, aprovechando las capacidades organizacionales . 


\section{Metodología}

En la presente investigación fue seleccionado el paradigma interpretativo o cualitativo, basado en un enfoque etnográfico, de carácter transversal, pues con dicha metodología se pueden estudiar los significados de las acciones humanas y de la vida social dentro de la realidad organizacional, la cual, en el presente estudio está referida al análisis del liderazgo ético en la gestión pública del municipio Mara del estado Zulia (Venezuela), a fin de comprenderla desde los significados de las personas implicadas, para estudiar sus creencias, intenciones, motivaciones y otras características del proceso de gestión, no observables directamente ni susceptibles de experimentación.

Adicionalmente, se selecciona como enfoque epistémico el introspectivo-vivencial, el cual, de acuerdo al criterio de Finol y Camacho (2006), surge de la interpretación de los símbolos científicos-sociales, mediante los cuales los actores de un determinado grupo social abordan la realidad, es decir, se interpreta la misma tal como aparece en el interior de los aspectos de conciencia subjetiva, de lo interno del investigador.

Aunado a esta técnica, se utiliza la entrevista, que es más íntima, flexible, además de abierta. Esta se define como una reunión para intercambiar información entre una persona (el entrevistador) y otra (el entrevistado). Para realizar dicha entrevista se diseñó un instrumento conformado por preguntas abiertas, que fue aplicado a la población de estudio, obteniendo con sus respuestas una comunicación directa que permitió la construcción conjunta de significados respecto al liderazgo transformacional que se lleva a cabo dentro de la Alcaldía de Mara del estado Zulia en Venezuela.

Para efectos de la presente investigación se selecciona el muestreo, específicamente, el intencional, no probabilístico, causal o incidental, de acuerdo a lo señalado por Parra (2006), con informantes clave, expertos en el área objeto de estudio, que laboran en las entidades públicas que conforman el Poder
Público Municipal de Mara, estado Zulia. En tal sentido, los informantes son cuatro (04): Representantes de la Función Ejecutiva, Función Deliberante, Función de Control Fiscal y la Función de Planificación, quienes son los directivos o líderes de las dependencias que tienen relación directa con el manejo de la parte administrativa, control y distribución de recursos públicos, que conforman los aspectos clave de análisis en el estudio que se lleva a cabo.

Seguidamente, se realiza la trascripción de dichas entrevistas a fin de presentar la evidencia referida a las manifestaciones de los informantes clave, la cual será presentada en tablas esquematizadas por cada entrevistado y por columnas donde se expondrá la categoría de entrada o la variable objeto de estudio, los códigos emergentes y el texto de la entrevista, siendo esto último las declaraciones textuales de cada entrevistado, de donde se extraerán las categorías emergentes que describen los aspectos fundamentales estudiados.

Con dichas categorías emergentes y la fundamentación teórica expuesta sobre el tema, se realiza la posterior triangulación, la cual, según Aguilar y Barroso (2015), es una técnica que permite combinar instrumentos de metodologías cuantitativa y cualitativas con la finalidad de obtener una mayor cantidad de evidencias y sustentaciones para ampliar el conocimiento del fenómeno estudiado.

\section{Resultados y discusión}

A continuación, se presentan los indicios etnográficos desde sus propios actores, por lo cual se hace la transcripción de las entrevistas realizadas, a fin de presentar la evidencia descrita y validada por las manifestaciones dadas por los informantes claves, con base en el guion de entrevista que fuere aplicado por parte del investigador a representantes de la Función Ejecutiva, Deliberante, de Control Fiscal y la Función de Planificación de la Alcaldía de Mara, buscando caracterizar, cómo a pesar de las diversas circunstancias que vive el país y que afectan a cada región y municipio, 
Villasmil, Milagros del C.; Romero, Francisco J. y Socorro, Cecilia C.

Liderazgo ético en la gestión pública municipal del estado Zulia, Venezuela

el alcalde Luis Caldera, mediante sus políticas de gestión ha influido en el desempeño de los funcionarios en las entidades administrativas a su cargo.

Cabe destacar, que el desarrollo de este apartado, forjó el análisis cualitativo como un momento heurístico flexible, orientado a un proceso en continuo avance, haciendo del análisis y la interpretación, los medios que permiten al investigador canalizar los ejes temáticos emergentes, releer sus notas de campo, inferir categorías, así como extraer conceptos y proposiciones, con el fin de darle sentido a los datos mediante la develación de sus significados.

En este sentido, de acuerdo al Cuadro 1, donde se muestran los resultados obtenidos del entrevistado 1, se manifiesta como la Alcaldía de Mara intenta la participación activa de los actores políticos, el trabajo educativo de formación, a través de talleres, la convivencia de los equipos de trabajo, los cuales deben asumir las normas imperantes, a la vez que la innovación, sobre lo cual afirma la necesidad de mecanismos tecnológicos que permitan el acercamiento de los líderes políticos, de los sujetos de gobierno al pueblo.

Análisis categorial-Informante 1. Liderazgo ético en la gestión municipal

\begin{tabular}{|c|c|c|}
\hline Categoría de entrada & Códigos emergentes & Texto de la entrevista \\
\hline $\begin{array}{c}\text { Procesos de transformación } \\
\text { organizacional }\end{array}$ & $\begin{array}{c}\text { Retos } \\
\text { Proyectos } \\
\text { Visión de país } \\
\text { Realidades } \\
\text { Mundo globalizado } \\
\text { Participación activa }\end{array}$ & $\begin{array}{l}\text { PREGUNTA: ¿Considera que usted fomenta las } \\
\text { transformaciones necesarias a la época actual? } \\
\text { RESPUESTA: "si porque uno de los grandes retos } \\
\text { que tiene la administración pública en América Latina } \\
\text { por supuesto en Venezuela mucho más, es el tema de } \\
\text { gobernar de acuerdo a las circunstancias teniendo claro } \\
\text { proyectos y visiones de país, estados y municipios pero } \\
\text { también no desvincularse de las realidades que se están } \\
\text { dando metabólicamente en el mundo, este es un mundo } \\
\text { totalmente globalizado, conectado por redes sociales, } \\
\text { interpretado con una participación activa del ciudadano, } \\
\text { cada día hay más participación activa del ciudadano en } \\
\text { sus diferentes expresiones pero la hay, entonces esto te } \\
\text { lleva a que tu tengas que ejercer el liderazgo ya con más } \\
\text { fuerza, para que tu generes corriente de pensamiento y } \\
\text { corrientes de acciones adecuados. }\end{array}$ \\
\hline
\end{tabular}

Fuente: Elaboración propia, 2020.

Asimismo, enfatiza el informante clave 1 que, ante los retos planteados a la administración pública, en este caso dirigida por el alcalde Luis Caldera, se deben generar proyectos de solución a las problemáticas sociales (seguridad, transporte, vivienda, salud, entre otras), asumiendo un liderazgo cercano, sin intermediarios, desde donde se refleje el pensamiento y la acción de gobierno, cambiando la adversidad por oportunidades, bajo los vestigios de responsabilidad de los actores implicados.
Contrastando los resultados del Cuadro 1 con la fundamentación teórica del estudio, se evidencia la discrepancia con respecto a lo planteado por Ferrer, et al. (2010), que el liderazgo ético, presenta diferentes alternativas estratégicas, centradas en participación y cooperación, en la construcción de pertinencia social institucional. En tal sentido, se debe elevar los niveles éticos sociales en la alcaldía de Mara; así como la formación de líderes éticos centrados en valores, capaces de fomentar una acción administrativa enfocada 
en relaciones de cooperación.

Con los factores mencionados anteriormente, puestos en práctica en la Alcaldía de estudio, deducen los investigadores que se generarán transformaciones que van a permitir que el talento humano se convierta en un agente de cambio, en el principal propulsor de los acontecimientos institucionales necesarios en la administración pública, pudiendo con ello revertir la ola de atropellos en contra de las necesidades colectivas, de la ciudadanía de Mara, a la cual, por el contrario, deben serle otorgados sus derechos y garantías constitucionales.

Con base a todo lo expuesto, es posible concebir al liderazgo ético como el estilo del funcionario para la acción gerencial, fundamentado en una actividad transformadora, derivada del pensamiento y la acción gerencial, donde se da lugar a la generación de cambios en la estructura y organización de la entidad pública, con miras al cumplimiento de normas para satisfacer el bienestar colectivo, tal como se visualiza en la Figura I.

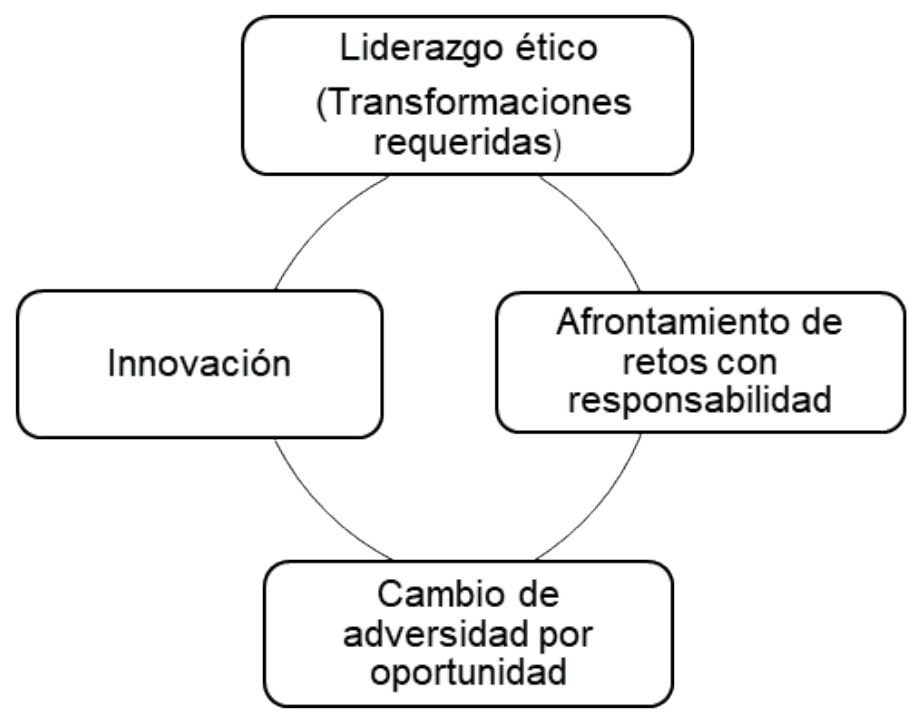

Fuente: Elaboración propia, 2020.

\section{Figura I: Estructura cognitiva entrevista No. 1}

Seguidamente, en la entrevista 2 suscitada en este estudio, manifiesta el entrevistado con gestos de espontaneidad y naturalidad, que en la Alcaldía de Mara se utilizan los documentos éticos propios de ese sector, tomando siempre previsiones para estar al día con las normas de uso interno a cumplir, buscando evadir las improvisaciones de la gestión efectuada. Asimismo, alude el informante, a la investigación de normativas, en pro de mejorar las propias, actualizando y haciendo las modificaciones necesarias para una mejor actuación dentro de la Alcaldía de Mara, con la finalidad de innovar en talento humano, en recursos materiales, sobre todo en la parte tecnológica, en la búsqueda de la simplificación de procedimientos administrativos y legales (ver Cuadro 2). 


\section{Cuadro 2}

\section{Análisis categorial-Informante 2. Liderazgo ético en la gestión municipal}

\begin{tabular}{|c|c|c|}
\hline $\begin{array}{c}\text { Categorías de } \\
\text { entrada }\end{array}$ & Códigos emergentes & Texto de la entrevista \\
\hline $\begin{array}{l}\text { Procesos de } \\
\text { transformación } \\
\text { organizacional }\end{array}$ & $\begin{array}{l}\text { Transformaciones } \\
\text { administrativas, } \\
\text { documentales, } \\
\text { tecnológicas } \\
\\
\text { Modernidad } \\
\text { Espontaneidad } \\
\text { Especificidad } \\
\text { Mejora } \\
\text { Procesos tecnológicos }\end{array}$ & $\begin{array}{l}\text { PREGUNTA: ¿Considera que usted fomenta las } \\
\text { transformaciones necesarias a la época actual? } \\
\text { RESPUESTA: "Dentro de las limitaciones que tenemos en } \\
\text { Venezuela, las transformaciones trascendentales en la parte } \\
\text { administrativa, documental, tecnológica si se hicieron en años atrás, } \\
\text { ahora un poco menos en la situación actual, pero si hemos hecho } \\
\text { transformaciones para responder a las exigencias del contexto } \\
\text { actual, tales como modificar un acuerdo de dos o tres páginas } \\
\text { para hacer algo moderno no tan burocrático, más espontáneo y } \\
\text { más específico del acuerdo que se va a hacer de diferentes puntos, } \\
\text { traspasos, documentos hemos mejorado ahí. También hemos } \\
\text { hecho transformaciones en grabaciones tratamos de hacerlo lo más } \\
\text { tecnológico posible, en la trascripción de documentos, pasamos de } \\
\text { la parte manual a la tecnológica y virtual. }\end{array}$ \\
\hline
\end{tabular}

Fuente: Elaboración propia, 2020.

Sin embargo, la gestión de la Alcaldía de Mara ha venido basándose en un trabajo conjunto, donde si se hace necesario, se realizan sesiones urgentes que permitan solucionar problemas de la colectividad, aprobando proyectos en busca de las transformaciones administrativas y tecnológicas, que den paso a la modernidad, a la actualización de estos tiempos, en aras de facilitar los procesos instaurados, incorporando la virtualidad en los mismos, a fin de agilizar los trámites municipales, especialmente, en la Alcaldía de Mara, que dadas las condiciones de escasos recursos que caracterizan la región, es preciso hacerles más fáciles y a su alcance la respuesta a sus requerimientos.

En relación con la fundamentación teórica del estudio, Garbanzo-Vargas (2015), destaca que cuando se busca gestionar los cambios en una organización, en este caso en la administración pública, se requiere que el talento humano apoye las transformaciones de modernidad y actualización necesarias, además de contar con un liderazgo efectivo, pudiendo inferirse con ello que así el funcionario público es capaz de canalizar la cultura de las organizaciones en los procesos de implementación de las acciones necesarias.

Adicionalmente, los investigadores advierten diferencia con el criterio de Mendonca y Kanungo (2009), el cual establece que la contribución que supone el ejercicio de la acción de un líder ético, en este caso en el desempeño de su cargo en la administración pública, provoca la transformación de los colaboradores en las tareas a ser implementadas en la gestión, sirviendo como referente y testimonio de vida a través de una conducta plena de virtudes así como valores personales y laborales.

Con base a todo lo expuesto, se concibe al liderazgo ético como un estilo de trabajo del funcionario público basado en la humanización, devenido de la normativa que rige su ejercicio y de la percepción de su propia praxis, canalizando las situaciones por atender con el fin de producir las transformaciones necesarias a la comunidad y la cultura de los pueblos en los cuales se inserta su acción gerencial, tal como muestra la Figura II. 


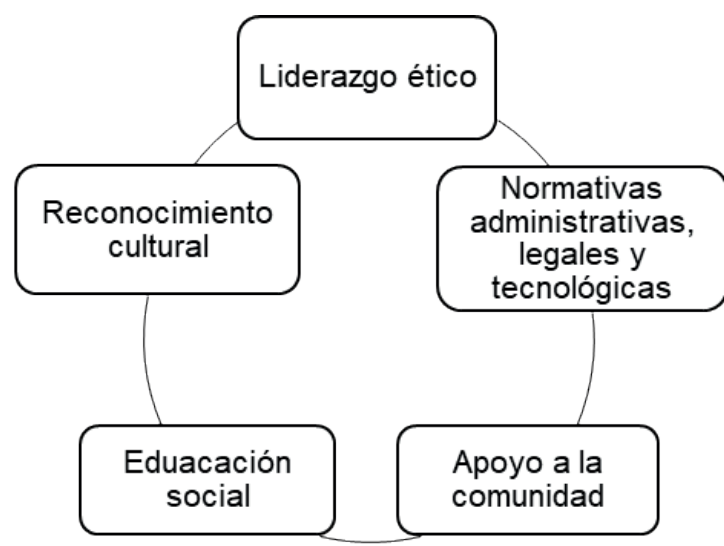

Fuente: Elaboración propia, 2020.

\section{Figura II: Estructura cognitiva entrevista No. 2}

Consecutivamente, en el Cuadro 3, manifiesta el entrevistado 3, de manera convencida, que, en la administración pública, lugar este donde se desempeña, se utilizan una serie de normas de control interno que son las que orientan la gestión a ser cumplida en la Alcaldía de Mara y las cuales se constituyen en un direccionamiento estratégico basado en los valores corporativos, la misión y la visión institucional.

\section{Cuadro 3}

\section{Análisis categorial-Informante 3. Liderazgo ético en la gestión municipal}

\begin{tabular}{|c|c|c|}
\hline $\begin{array}{c}\text { Categorías de } \\
\text { entrada }\end{array}$ & $\begin{array}{c}\text { Códigos } \\
\text { emergentes }\end{array}$ & Texto de la entrevista \\
\hline $\begin{array}{c}\text { Procesos de } \\
\text { transformación } \\
\text { organizacional }\end{array}$ & $\begin{array}{l}\text { Generación de } \\
\text { transformaciones } \\
\text { Actualización } \\
\text { Modernidad } \\
\text { Adaptación a los } \\
\text { tiempos } \\
\text { Fortalecimiento } \\
\text { institucional } \\
\text { Adaptación } \\
\text { a normas } \\
\text { internacionales } \\
\text { Transformación } \\
\text { de las estructuras } \\
\text { de gobierno } \\
\text { Inspiración } \\
\text { Innovación }\end{array}$ & $\begin{array}{l}\text { PREGUNTA: ¿Considera que usted fomenta las transformaciones } \\
\text { necesarias a la época actual? } \\
\text { RESPUESTA: "fundamentalmente es necesario generar las transformaciones } \\
\text { porque si no generamos esas transformaciones nos vamos a convertir en } \\
\text { un órgano si se quiere anacrónico, estancado en el tiempo y ahora que nos } \\
\text { encontramos en un mundo donde cada día debemos ser más actualizados } \\
\text { a la modernidad porque en la medida que nosotros podamos modernizar } \\
\text { estos aspectos de la gestión y adaptarnos a la época actual, nos estamos } \\
\text { fortaleciendo institucionalmente a la administración pública, en este caso a } \\
\text { la gestión de la Contraloría debemos adaptarnos a las transformaciones que } \\
\text { involucran un mundo globalizado, por lo menos en los aspectos necesarios a } \\
\text { algunas actividades que se realizan, por lo menos las auditorias que son si se } \\
\text { quiere uno de los procesos medulares que desarrolla la Contraloría, debemos } \\
\text { estar adaptados a las normas internacionales de auditoria interna a través de } \\
\text { los lineamientos de la Contraloría, debemos adaptarnos porque no estamos } \\
\text { aislados en el mundo globalizado, debemos transformar las estructuras } \\
\text { de gobierno, de gestión deben ser transformadas a la luz de esas nuevas } \\
\text { inspiraciones que genera un mundo globalizado, porque si no se logra esa } \\
\text { transformación quedaríamos desfasados y de manera anacrónica pudiéramos } \\
\text { nosotros desarrollar nuestra gestión, nos aislaríamos completamente, es } \\
\text { necesario y fundamental innovar el proceso de transformación". }\end{array}$ \\
\hline
\end{tabular}

Fuente: Elaboración propia, 2020. 
Villasmil, Milagros del C.; Romero, Francisco J. y Socorro, Cecilia C.

Liderazgo ético en la gestión pública municipal del estado Zulia, Venezuela

Desde la misma perspectiva, el informante expresa la necesidad de la actualización en la gestión pública, la adhesión a la modernidad, la adaptación a los tiempos, pero desde la óptica del fortalecimiento institucional, e incluso adaptándose a las normas internacionales, lo cual exige la transformación de las actuales estructuras de gobierno, considerando la propia inspiración del talento humano, con base en la innovación de la época.

Los planteamientos hechos por el entrevistado 3, no son coincidentes con el criterio de Guiaiquirima y Seijo (2010), quienes plantean que en el seno de las organizaciones humanas se hace necesaria la recuperación de los valores éticos como referentes de su actuación. De allí, que pueden inferir los investigadores que las estructuras económicas, políticas y sociales, así como las de gobierno en la administración pública, deban ser modificadas, pasando a ser instrumentos al servicio del hombre, debiendo promover a través de un liderazgo sustentado en valores éticos, los derechos fundamentales sobre las bases de un ambiente con calidad ética, dentro del marco de la legalidad que garantice un servicio público acorde con las exigencias de los ciudadanos en los albores del siglo XXI.

Con base a todo lo expuesto, se concibe al liderazgo ético como la conducta ejemplar del funcionario público, basada en las normas de control interno y en lineamientos estratégicos que rigen en la Alcaldía de Mara, llevándolos a la transparencia en sus acciones, para la mejora y desarrollo de la gestión pública hacia la competitividad, permitiendo lograr las transformaciones necesarias en las estructuras de gobierno en aras del fortalecimiento ético institucional, dirigido por un liderazgo conjunto basado en nuevas tendencias organizacionales, tal como se muestra en la Figura III.

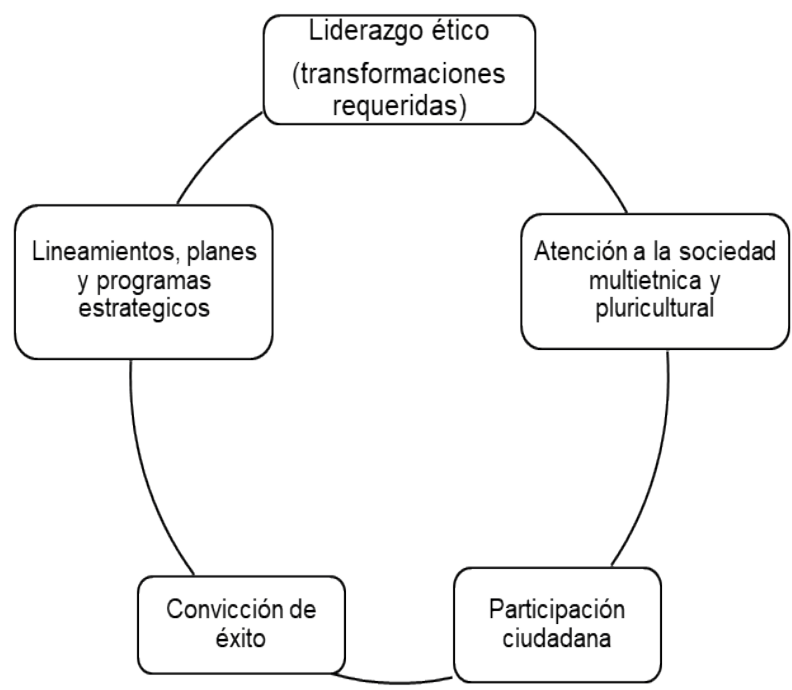

Fuente: Elaboración propia, 2020.

Figura III: Estructura cognitiva entrevista No. 3 
Ahora bien, el informante clave en la entrevista 4 realizada, enfatiza que, si bien en la mayoría de los casos se rigen por las leyes, las ordenanzas propias de la entidad pública municipal en estudio, la Alcaldía de Mara, igualmente en ocasiones debe improvisar para poder solventar las situaciones que se le presentan cotidianamente, tal como se aprecia en el Cuadro 4.

\section{Cuadro 4}

Análisis categorial: Liderazgo ético en la gestión municipal

\begin{tabular}{cll} 
Categorías de entrada & Códigos emergentes & \multicolumn{1}{c}{ Texto de la entrevista } \\
\hline $\begin{array}{c}\text { Procesos de transformación } \\
\text { organizacional }\end{array}$ & Participación conjunta & $\begin{array}{l}\text { PREGUNTA ¿Considera que usted fomenta las } \\
\text { transformaciones necesarias a la época actual? } \\
\text { RESPUESTA: Si las transformamos pero la hacemos } \\
\text { en forma conjunta tomando la participación de cada } \\
\text { uno de ellos y a través de esa participación que hacen } \\
\text { conjuntamente todos nosotros tomamos decisiones en } \\
\text { presencia de ellos" }\end{array}$ \\
Toma de decisiones
\end{tabular}

Fuente: Elaboración propia (2019).

De la misma manera, el informante arguye que la capacidad de respuesta debe poseerse en todo momento, para poder concretar los logros, no obstante, se debe encaminar la gestión pública hacia la responsabilidad, la honestidad y la humildad, con cuyos conceptos serían posibles las transformaciones en esa entidad, lográndose incluso la ausencia de corrupción en el nivel político.

Se desprende asimismo de las manifestaciones del informante que, en la Alcaldía de Mara, si bien existe la intención de atender a todas las solicitudes introducidas, a veces no se acuerdan inversiones, por lo cual hay sectores de las comunidades sin obras, lo cual genera inquietudes para provocar la escucha, el avance social, la participación protagónica del pueblo en la toma de decisiones.

De la misma manera, los resultados confirman la teoría de Pérez (2012), al afirmar que en algunas organizaciones aún no se perfila un estilo de liderazgo definido, ni más específicamente el estilo ético, necesario en las entidades propias de la administración pública, lo cual en el criterio del investigador redunda en una falla gerencial, toda vez que en estas entidades oficiales debe aludirse a la generación de una serie de competencias en los sujetos que apuntalen el cambio organizacional, produciendo transformaciones hacia el bienestar físico y mental de la sociedad.

Por lo tanto, es necesario que estas instituciones tengan definidos unos lineamientos, que les permitan identificarse con todos sus miembros, cuyos parámetros, no solo sean conocidos por todos los miembros de la organización, sino que además hayan participado en su elaboración. Lo cual, obliga a los directivos a repensar, cuáles son los documentos que son realmente necesarios implementar para contribuir a la imagen y reputación de la institución, sin llegar a atentar contra el bienestar general de la misma.

Con base en todo lo expuesto, se concibe al liderazgo ético como la tarea del funcionario público de dar cumplimiento estricto a las normas internas de la entidad en estudio, para el logro de la capacidad de respuesta ante las necesidades colectivas, pudiendo generar un plan de acción basado en las inversiones para los sectores necesitados, de acuerdo a la agenda 
Villasmil, Milagros del C.; Romero, Francisco J. y Socorro, Cecilia C.

Liderazgo ético en la gestión pública municipal del estado Zulia, Venezuela

diseñada, y producir las transformaciones que

Figura IV.

requiere el Municipio, como se muestra en la

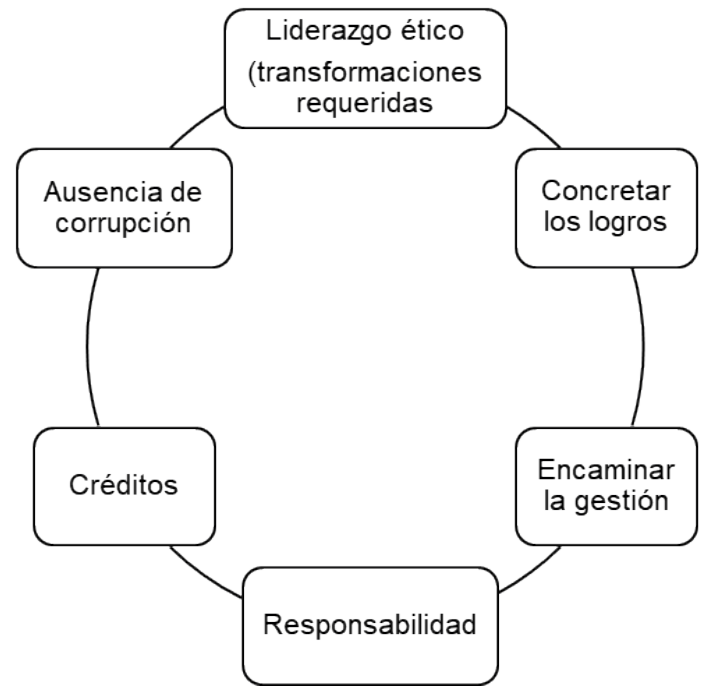

Fuente: Elaboración propia, 2020.

Figura IV: Estructura cognitiva entrevista No. 4

Con todo lo presentado, es posible advertir que el liderazgo ético como factor potenciador de la transformación organizacional en la entidad municipal Alcaldía de Mara, se convierte en un mecanismo expedito en el funcionario público, para ser ejecutor de las decisiones acordadas por los líderes referentes, pudiendo en tal sentido convertirse en un agente de cambio en la gestión oficial, de Estado, en un agente transformador, derivando como resultado la satisfacción de los usuarios del servicio y la resolución de problemas en las comunidades en las cuales insertan sus acciones y políticas públicas.
Con el fin de finiquitar el proceso de teorización, se implementó la triangulación, entre los aportes recabados de los entrevistados y conceptos sensibilizadores con la agudeza del investigador. Donde, las categorías y subcategorías identificadas fueron re-contextualizadas y transformadas en ideas significativas, que englobaron los significados de los actores sociales involucrados con los del investigador. Significados que se compararon para construir la teoría sustantiva al momento de refinarla, dándole formalidad. A continuación, se explican los elementos constitutivos del constructo elaborado, apreciados en la siguiente Figura V. 


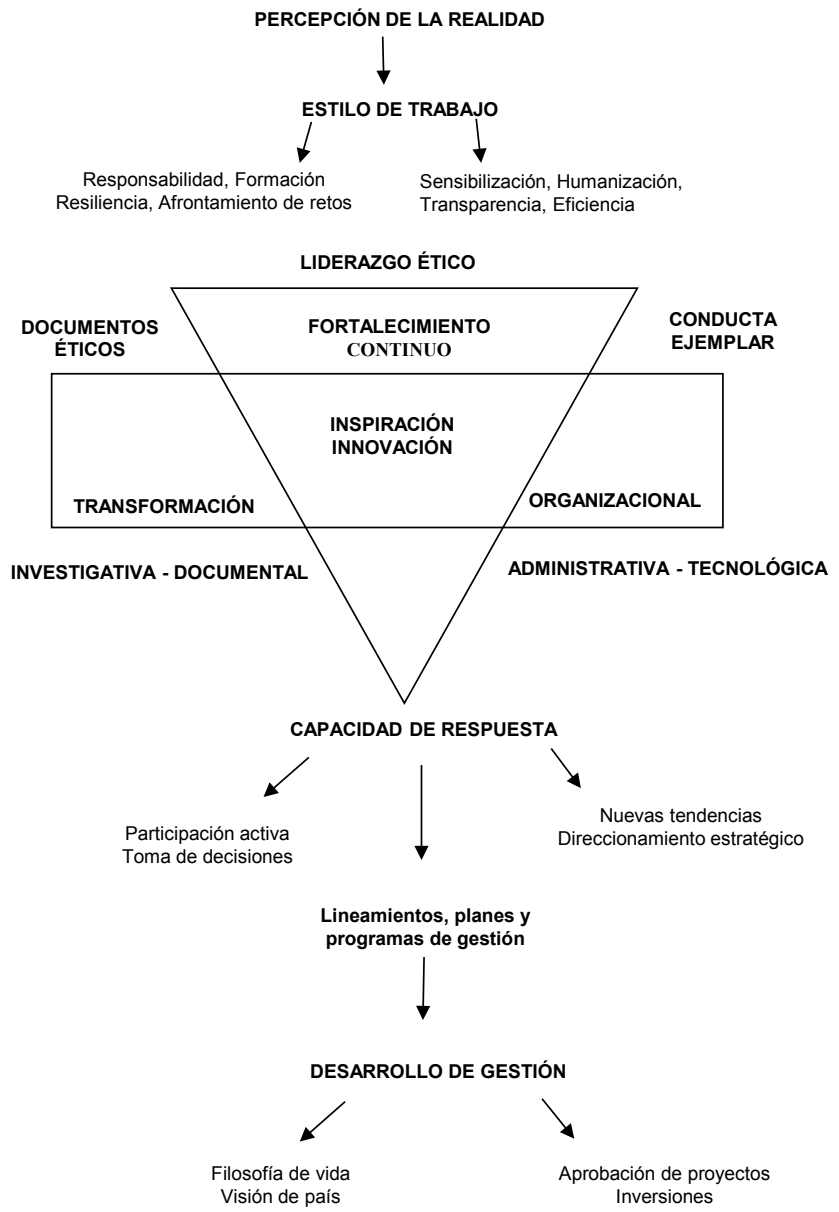

Fuente: Elaboración propia, 2020.

Figura V: Constructo teórico concebido en la investigación

\section{Conclusiones}

Luego del estudio realizado se evidenció como influye el liderazgo en los procesos de transformación de la gestión pública municipal, los cuales conforme a los fundamentos teóricos deben estar referidos a motivar, alistar a los individuos o el grupo, reorganizándolos para que acepten la necesidad de cambiar sus estrategias de trabajo, su entorno organizacional, produciendo alteraciones estructurales, de comportamiento, generando 
Villasmil, Milagros del C.; Romero, Francisco J. y Socorro, Cecilia C.

Liderazgo ético en la gestión pública municipal del estado Zulia, Venezuela

que se estabilicen los cambios interiorizados y ejecutados.

No obstante lo planteado, en las instituciones públicas del poder público municipal de Mara, a pesar de los esfuerzos de cambios, se adolece de algunas limitaciones para el logro de las transformaciones desde el punto de vista político, jurídico, económico, social, tecnológico, que le permita constituirse en un referente positivo de la administración pública local $\mathrm{y}$, constituir un nicho productivo desde donde sea posible establecer parámetros de comparación con otras entidades del sector oficial. Por lo cual, es preciso considerar para esto los planteamientos de Díaz (2011), quien señala que la ética dentro de la gestión pública, representa un instrumento estratégico que facilita la toma de decisiones correctas, asegurando la confiabilidad de los gobiernos en el mundo global que exige cada vez más integridad y compromiso, responsabilidad $\mathrm{y}$ respeto con el ser humano y con el medio ambiente.

Adicionalmente, se conoció que se han intentado desarrollar cambios socio-culturales, vale decir, la modificación de actitudes, comportamientos, valores, creencias, hábitos, ritos, mitos, símbolos, lenguaje, intereses comunes y mecanismos de participación, como métodos para lograr satisfacción y armonía en el ambiente laboral, pero la modificación de la cultura organizacional debe ser canalizada de manera metódica, induciendo a los involucrados, a través de talleres grupales, a un cambio gradual progresivo en los componentes cognitivo y actitudinal de la personalidad, además de propiciar el sentido de identificación y pertenencia institucional para el logro de un mejor desempeño en la administración pública.

Cabe destacar, que la participación ciudadana en la agenda pública, se convierte hoy en un desafío institucional, debiendo promover la participación activa y la toma de decisiones de los usuarios del sistema municipal, a la vista de las nuevas tendencias organizacionales del mundo y basados en procesos alusivos al direccionamiento estratégico, desde donde se visione la organización para la eficiencia y la optimización de sus procedimientos.

Todo lo antes mencionado, permite a las organizaciones modernas, producir la necesaria transformación organizacional, medianamente inexistente en algunas oficinas examinadas que integran los órganos del poder público de Mara, a fin de que su gestión investigativa, normativa, tecnológica, administrativa, sea la más idónea a los intereses colectivos y de fiel cumplimiento a los fines del propio Estado, quien asume la obligación de establecer las acciones, medidas y políticas públicas que coadyuven al bienestar de los ciudadanos.

\section{Referencias bibliográficas}

Aguilar, S., y Barroso, J. (2015). La triangulación de datos como estrategia en investigación educativa. Revista de Medios y Educación, (47), 73$88 . \quad$ https://doi.org/10.12795/ pixelbit.2015.147.05

Alvis, R., Alvis, C. R., y Alvis, L. (2019). Liderazgo político protagónico de la mujer en procesos comunitarios el Cesar, Colombia. Revista Opción, 35(89-2), 1235-1265.

Bass, B. M., y Bass, R. (2008). The bass handbook of leadership: Theory, research, and managerial applications. Editorial Free Press.

Brown, M. E., y Treviño, L. K. (2006). Ethical leadership: A review and future directions. The Leadership Quarterly, 17(6), 595-616. https://doi. org/10.1016/j.leaqua.2006.10.004

Calderón, G., Cuartas, J., y Álvarez, C. M. (2009). Transformación organizacional y prácticas innovadoras de gestión humana. Universidad Nacional de Colombia. Revista Innovar, 19(35), 151-166.

Correa, J. S., Rodríguez, M. D. P., y Pantoja, M. A. (2018). Liderazgo ético en 
las organizaciones: Una revisión de la literatura. AD-minister, (32). 57-82. $\quad$ https://doi.org/10.17230/administer.32.3

Díaz, J. F. (2011). El arte de tomar decisiones con contenidos éticos. Revista Venezolana de Gerencia, 16(54), 323-325.

Espinal, E. (2017). Necesidad de reflexionar la vinculación entre ética y política en los albores del nuevo milenio. Revista de Administración Pública, LII(3), 171-187. https://inap.mx/wp-content/ uploads/2020/08/rap144.pdf

Fernández, M. C., y Quintero, N. (2017). Liderazgo transformacional $\mathrm{y}$ transaccional en emprendedores venezolanos. Revista Venezolana de Gerencia, 22(77), 56-74.

Ferrer, J., Colmenares, F., y Clemenza, C. (2010). Un líder ético para el cambio: plataforma de gestión estratégica en Instituciones Universitarias. Revista de Ciencias Sociales (Ve), XVI(4), 642653.

Finol, M., y Camacho, H. (2006). El proceso de investigación cientifica. Editorial Ediluz.

Font, I., Gudiño, P., Sánchez, A., Córdova, A. O., y Morales, A. (1999). El administrador frente a la necesidad de transformación organizacional. Revista Gestión y Estrategia, (15), 67-75.

Garbanzo-Vargas, G. M. (2015). Desarrollo organizacional y los procesos de cambio en las instituciones educativas, un reto de la gestión de la educación. Revista Educación, 40(1), 67-87. http://dx.doi. org/10.15517/revedu.v40i1.22534

González, N., y Rada, N. (2017). Estrategias para la potenciación de líderes transformacionales juveniles. Revista de Ciencias Sociales (Ve), XXIII(1), 81-89.

Guaiquirima, C., y Seijo, C. (2010). El liderazgo ético: un constructo ambiguo en la administración pública. Editorial ASTRO Data.

Loaiza, C., y Pirela, L. (2015). Liderazgo en organizaciones venezolanas. Revista Venezolana de Gerencia, 20(69), 152171.

Mendonca, M., y Kanungo, R. N. (2009). Ethical leadership (work and organizational psychology). Open University Press.

Mendoza, E., y Mendoza, M. A. (2008). El liderazgo ético en organizaciones postmodernas. Revista de Artes y Humanidades UNICA, 9(22), 59-78.

Narváez, M., Ferrer, J., Fernández, G., y Senior, A (2009). Rasgos del componente ético en el cumplimiento de la gestión empresarial social de la pequeña y mediana empresa de la región Paraguaná. Revista de Ciencias Sociales (Ve), XV(1), 112-125. https:// doi.org/10.31876/rcs.v15i1.25431

Nava, D., Seijo, C., y Mier, C. (2014). Inteligencias múltiples: característica congruente del liderazgo ético en entidades financieras. Praxis, 10(1), 91-99. https://doi. org/10.21676/23897856.1361

Parra, J. (2006). Guía del muestreo. Colección XLVIII Aniversario FCES. Dirección de Cultura de la Universidad del Zulia.

Paz, A., Núñez, M. G., García, J., y Salom, J. (2016). Rol del liderazgo ético en organizaciones académicas. Revista Opción, 32(E-12), 148-168.

Pedraja-Rejas, L., Rodríguez-Ponce, E., y Rodríguez-Ponce, J. (2008). Importancia de los estilos de liderazgo sobre la eficacia: Un estudio comparativo entre grandes y pequeñas y medianas empresas privadas. Revista de Ciencias Sociales (Ve), XIV(1), 20-29. https://doi.org/10.31876/rcs. $\underline{\mathrm{v} 14 \mathrm{i} 1.25384}$ 
Villasmil, Milagros del C.; Romero, Francisco J. y Socorro, Cecilia C.

Liderazgo ético en la gestión pública municipal del estado Zulia, Venezuela

Pérez, F. (2012). Competencias de liderazgo transformacional subyacentes en los directores de las Instituciones de Educación Básica de la Parroquia Escuque. Revista Arbitrada del Centro de Investigación y Estudios Gerenciales, 2(4), 1-19.

Petit, E. (2012). El desarrollo organizacional innovador: un cambio conceptual para promover el desarrollo. Revista de Ciencias Sociales (Ve), XVIII(1), 74-88. https://doi.org/10.31876/rcs. v18i1.24963

Ramírez, M. (2012). Estilos de liderazgo $\mathrm{y}$ sus enfoques gerenciales. Una aproximación teórica-metodológica para el análisis de la dirección organizacional. Revista de Ciencias Sociales (Ve), XVIII(1), 89-98.

Romero, G. E. (2006). Principios y valores éticos en las organizaciones bancarias respecto a la calidad del servicio y atención al cliente. Revista Venezolana de Gerencia, 11(36), 638-651.

Vegas, H. (2016). La teoría fundamentada como herramienta metodológica para el estudio de la gestión pública local. Revista Venezolana de Gerencia, 21(75). 413-426.

Villasmil, M., Alvarado, L., y Socorro, C. (2019). Responsabilidad por ilícitos y sanciones en el código orgánico tributario venezolano. Revista Venezolana de Gerencia, 24(2), 270284. https://doi.org/10.37960/revista. v24i2.31493

Villasmil-Molero, M., Alvarado-Peña, L., Socorro-González, C., y GamboaCaicedo, N. (2018). Contribuciones parafiscales: Factor determinante para la seguridad social. Revista de Ciencias Sociales (Ve), XXIV(3). 21-35. 